Publ. RIMS, Kyoto Univ.

18 (1982), 101-117

\title{
A Posteriori Componentwise Error Estimate for a Computed Solution of a System of Linear Equations
}

By

Tetsuro YAMAMOTO*

\section{Introduction}

Let $x^{(0)}=\left(x_{1}^{(0)}, \cdots, x_{n}^{(0)}\right)^{t}$ be a computed solution of a system of $n$ linear equations

$$
A x=b
$$

where $A=\left(a_{i j}\right), x=\left(x_{1}, \cdots, x_{n}\right)^{t}$ and $b=\left(b_{1}, \cdots, b_{n}\right)^{t}$. Then a question naturally arises as to whether the approximale solution $x^{(0)}$ is a satisfactory one. Let $A$ be nonsingular and $L$ be an approximation for the inverse of $A$. In practical computation, $L$ may be chosen as a computer result for $A^{-1}$. Let $R=I_{n}-L A$ and $r=A x^{(0)}-b$ where $I_{n}$ denotes the $n \times n$ identity. If $R$ has the spectral radius which is smaller than one, then $L$ is nonsingular and

$$
A^{-1}=\left(I_{n}-R\right)^{-1} L .
$$

Hence, if we denote by $x^{*}$ the exact solution of $(0.1)$, then we have

$$
x^{*}-x^{(0)}=-A^{-1} r=-\left(I_{n}-R\right)^{-1} L r,
$$

or

$$
\left\|x^{*}-x^{(0)}\right\| \leqq\left\|\left(I_{n}-R\right)^{-1}\right\| \cdot\|L r\| \leqq(1-\|R\|)^{-1}\|L r\|
$$

with some vector norm $\|\cdot\|$, provided that $\|R\|<1$. Therefore, if $\|L r\|$ and $\|R\|$ are small enough, then we can conclude from (0.3) that $x^{(0)}$ is accurate. However, if there are large and small values among $\left|x_{1}^{(0)}\right|, \cdots$,

Communicated by S. Hitotumatu, December 22, 1980.

* Department of Mathematics, Faculty of Science, Ehime University, Matsuyama 790, Japan. 
$\left|x_{n}{ }^{(0)}\right|$, then (0.3) does not give a sharp estimate for a specified component of $x^{(0)}$. Therefore, in such a case, the use of $(0.2)$ is desirable. However, (0.2) requires the computation of the inverse of $I_{n}-R$, which is troublesome.

In this paper, we shall first prove a result for finding the componentwise error bounds of $x^{(0)}$ without using $\left(I_{n}-R\right)^{-1}$. Next, we shall perform its error analysis for a machine having a floating-point arithmetic device with the base $\beta$ in which the results are chopped to $t \beta$-digits. The results of the analysis show that our method works well if $\|R\|_{\infty}$ $<1$ and

$$
\|L\|_{\infty}\left(\|A\|_{\infty}+\|A\|_{\infty} \cdot\left\|x^{(0)}\right\|_{\infty}+\|r\|_{\infty}\right) n \beta^{1-\iota}
$$

is not large, where $\|\cdot\|_{\infty}$ denotes the maximum norm. Further, based on this result, we shall propose a practical algorithm for estimating rigorously the error of $x^{(0)}$. Finally, numerical examples are given, which illustrate our results.

\section{$\S$ 1. Notation}

Throughout this paper, we shall use the following notation (cf. Urabe [5] and Yamamoto [8]): Let $x=\left(x_{1}, \cdots, x_{n}\right)^{t}$ and $y=\left(y_{1}, \cdots, y_{n}\right)^{t}$ be two vectors. Then we write $x \geqslant y$ or $y \leqslant x$ if $x_{i} \geqq y_{i}$ for all $i$. We put $\nu[x]=\left(\left|x_{1}\right|, \cdots,\left|x_{n}\right|\right)^{t}$. The same notation is used for matrices $A$ $=\left(a_{i j}\right)$ and $B=\left(b_{i j}\right): A \geqslant B$ or $B \preccurlyeq A$ if $a_{i j} \geqq b_{i j}$ for all $i, j$ and we put $\nu[A]=\left(\left|a_{i j}\right|\right)$.

\section{§2. A Result}

Let $x^{(0)}$ be an approximate solution of the system $(0.1)$ which has the unique solution $x^{*}$ and $L$ be an approximation for the inverse of $A$. We put $K=\nu\left[I_{n}-L A\right]$. Then we have the following theorem.

Theorem 1. Let $\|\cdot\|$ be a monotonic vector norm and $\kappa$ be a vector such that

$$
K x \leqslant\|. x\| \kappa
$$


for all $x \geqslant 0$. We assume that $\|\kappa\|<1$ and put

$$
\varepsilon=\nu\left[L\left(A x^{(0)}-b\right)\right], a=(1-\|\kappa\|)^{-1}\|\varepsilon\| \text { and } \alpha=\varepsilon+a \kappa \text {. }
$$

Then we have

$$
\nu\left[x^{*}-x^{(0)}\right] \preccurlyeq \alpha .
$$

Further, if we define a sequence of vectors $\left\{\alpha^{(k)}\right\}$ by

$$
\alpha^{(0)}=\alpha, \quad \alpha^{(k+1)}=\varepsilon+K \alpha^{(k)}, \quad k=0,1,2, \cdots,
$$

then

$$
\alpha^{(0)} \geqslant \alpha^{(1)} \geqslant \cdots \rightarrow \alpha^{*}=\left(I_{n}-K\right)^{-1} \varepsilon=\left(\alpha_{1}^{*}, \cdots, \alpha_{n}^{*}\right)^{t}
$$

and

$$
\nu\left[x^{*}-x^{(0)}\right] \preccurlyeq \alpha^{*} \preccurlyeq \alpha^{(k)}, \quad k \geqq 0 .
$$

That is, we have

$$
\left|x_{i}^{*}-x_{i}^{(0)}\right| \leqq \alpha_{i}^{*} \leqq \alpha_{i}^{(k)}, \quad 1 \leqq i \leqq n,
$$

for every $k \geqq 0$, where $x_{i}^{*}, x_{i}^{(0)}$ and $\alpha_{i}^{(k)}$ clenote the $i$-th component of $x^{*}, x^{(0)}$ and $\alpha^{(k)}$, respectively'.

Proof. We first remark that $\|K\|<1$, because the norm is monotonic and (2.1) implies $\|K x\| \leqq\|x\| \cdot\|\kappa\|<\|x\|$ for $x \neq 0$. Therefore, it follows from (0.2) that

$$
\begin{aligned}
\nu\left[x^{*}-x^{(0)}\right] & \leqslant \nu\left[\left(I_{n}-R\right)^{-1}\right] \nu[L r] \\
& =\nu\left[I_{n}+R+R^{2}+\cdots\right] \varepsilon \\
& \preccurlyeq\left(I_{n}+\nu[R]+\nu\left[R^{2}\right]+\cdots\right) \varepsilon \\
& \leqslant\left(I_{n}+K+K^{2}+\cdots\right) \varepsilon \\
& \leqslant \varepsilon+\|\varepsilon\| \kappa+\|K \varepsilon\| \kappa+\cdots \\
& \leqslant \varepsilon+\left(\|\varepsilon\|+\|\varepsilon\| \cdot\|\kappa\|+\|\varepsilon\| \cdot\|\kappa\|^{2}+\cdots\right) \kappa \\
& =\varepsilon+a \kappa=\alpha .
\end{aligned}
$$

Next, the monotone decreasing property of the sequence $\left\{\alpha^{(k)}\right\}$ is proved by induction on $k$ : In fact, by noting that

$$
\|\alpha\| \leqq\|\varepsilon\|+a\|\kappa\|=a,
$$


we have

$$
\alpha^{(1)}=\varepsilon+K \alpha \preccurlyeq \varepsilon+\|\alpha\| \kappa \preccurlyeq \varepsilon+a \kappa=\alpha=\alpha^{(0)}
$$

and $\alpha^{(k)} \preccurlyeq \alpha^{(k-1)}$ implies that

$$
\alpha^{(k+1)}=\varepsilon+K \alpha^{(k)} \preccurlyeq \varepsilon+K \alpha^{(k-1)}=\alpha^{(k)} .
$$

Therefore, $\left\{\alpha^{(k)}\right\}$ converges to a vector $\alpha^{*} \geqslant 0$, which satisfies

$$
\alpha^{*}=\varepsilon+K \alpha^{*} .
$$

It follows from this that

$$
\alpha^{*}=\left(I_{n}-K\right)^{-1} \varepsilon=\left(I_{n}+K+K^{2}+\cdots\right) \varepsilon .
$$

Consequently we obtain from (2.3)

$$
\nu\left[x^{*}-x^{(0)}\right] \preccurlyeq \alpha^{*} \preccurlyeq \cdots \preccurlyeq \alpha^{(k)} \preccurlyeq \cdots \preccurlyeq \alpha^{(1)} \preccurlyeq \alpha^{(0)}=\alpha .
$$

Q.E.D.

Remark. For the maximum norm $\|\cdot\|_{\infty}$, the $i$-th component $\kappa_{i}$ of the vector $\kappa$ is given by

$$
\kappa_{i}=\sum_{j=1}^{n} \kappa_{i j}
$$

where $\kappa_{i j}$ denote the $(i, j)$ elements of the matrix $K$. Hence, in this case, we have $\|\kappa\|_{\infty}=\|K\|_{\infty}$.

\section{§ 3. Floating-Point Error Analysis}

In practice, we cannot obtain the exact values of the vectors $\alpha^{(k)}(k$ $\geqq 0$ ), because of the rounding errors made in the computation. So the floating-point error analysis would be necessary. We shall call it out for the result of Theorem 1 by choosing the maximum norm $\|\cdot\|_{\infty}$. We assume that we work with a computer in which numbers are represented in the form $\pm d \beta^{m}$ where $\beta$ is the base of the number system and $d$ is the mantissa consisting of $t$ digits and $0 \leqq d<1$. We use the techniques due to Wilkinson [6], [7], Forsythe and Moler [2] and Paige [3]. Thus, if $\circ$ denotes any of the four arithmetic operations,,$+- \times, /$, then $a=\mathrm{fl}(b \circ c)$ means that $a, b$ and $c$ are floating-point numbers and $a$ is obtained from $b$ and $c$ using the appropriate floating-point operation. 
We assume that $n \geqq 2$,

$$
A(a \circ b)=a \circ b(1+\xi), \quad|\xi|<\beta^{1-t},
$$

and

$$
1.006(n+1) \beta^{1-t}<0.01
$$

Note that (3.1) reflects a machine in which the results are chopped to $t \beta$-digits. If we consider a machine in which the results are rounded to $t \beta$-digits, then we should replace $\beta^{1-t}$ in $(3.1)$ and $(3.2)$ by $2^{-1} \beta^{1-t}$, and the inequality $<$ in $(3.1)$ by $\leqq$. Observe also that $(3.2)$ means that $\beta^{1-t}<0.01 / 3.018<0.0034$.

In the following, for the sake of convenience, we shall write $\theta_{n}$ $=n \beta^{1-t}$ and use the following inequalities:

(3.3) If $0 \leqq n a<0.01$, then $(1+a)^{n} \leqq e^{n a}<1+1.006 n a$.

The following two lemmas are essentially proved in Wilkinson [6].

Lemma I. If $a_{i}, i=1,2, \cdots, n$ are the floating-point numbers, then

$$
\mathrm{fl}\left(a_{1}+\cdots+a_{n}\right)=\sum_{i=1}^{n} a_{i}\left(1+\xi_{i}\right)
$$

rohere

$$
1+\hat{\xi}_{i}= \begin{cases}\left(1+\eta_{2}\right) \cdots\left(1+\eta_{n}\right) & (i=1) \\ \left(1+\eta_{i}\right) \cdots\left(1+\eta_{n}\right) & (2 \leqq i \leqq n), \quad\left|\eta_{j}\right|<\beta^{1-\ell} \quad(1 \leqq j \leqq n) .\end{cases}
$$

Furthermore

$$
\mathrm{fl}\left|\left(a_{1}+\cdots+a_{n}\right)-\sum_{i=1}^{n} a_{i}\right|<1.006 \theta_{n-1} \sum_{i=1}^{n} a_{i}
$$

If $a_{i} \geqq 0 \quad(1 \leqq i \leqq n)$, then

$$
\sum_{i=1}^{n} a_{i}<\left(1-1.006 \theta_{n-1}\right)^{-1} \mathrm{~A}\left(a_{1}+\cdots+a_{n}\right) .
$$

Proof. The equality (3.4) is proved by induction on $n$. (3.6) follows from (3.4) since (3.3) and (3.5) imply that

$$
1+\left|\xi_{i}\right|<\left(1+\theta_{1}\right)^{n-1} \quad(1 \leqq i \leqq n)
$$

Q.E.D. 
Lemma 2. If $a_{i}$ and $b_{i}$ are the floating-point numbers, then

$$
\mathrm{A}\left(a_{1} b_{1}+\cdots+a_{n} b_{n}\right)=\sum_{i=1}^{n} a_{i} b_{i}\left(1+\xi_{i}\right)
$$

where

$$
1+\xi_{i}= \begin{cases}\left(1+\eta_{1}\right)\left(1+\zeta_{2}\right) \cdots\left(1+\zeta_{n}\right) & (i=1) \\ \left(1+\eta_{i}\right)\left(1+\zeta_{i}\right) \cdots\left(1+\zeta_{n}\right) & (2 \leqq i \leqq n)\end{cases}
$$

with $\left|\eta_{i}\right|,\left|\zeta_{j}\right|<\beta^{1-t}, i=1,2, \cdots, n, j=2, \cdots, n$. Hence

$$
\left|\mathrm{fl}\left(a_{1} b_{1}+\cdots+a_{n} b_{n}\right)-\sum_{i=1}^{n} a_{i} b_{i}\right|<1.006 \theta_{n} \sum_{i=1}^{n} a_{i} b_{i},
$$

so that, if $a_{i} b_{i} \geqq 0$, then

$$
\sum_{i=1}^{n} a_{i} b_{i}<\left(1-1.006 \theta_{n}\right)^{-1} \mathrm{fl}\left(a_{1} b_{1}+\cdots+a_{n} b_{n}\right) .
$$

In the following, we denote by $\widetilde{a}$ the computer result for an expression $a$. Thus, if $a$ is a number, then $\widetilde{a}$ means the floating-point representation of $a$ in the machine. For simplicity, we assume that $\widetilde{x}^{(0)}$ $=x^{(0)}, \tilde{A}=A, \tilde{b}=b$ and $\tilde{L}=L$.

Lemma 3. Let $r=A x^{(0)}-b$ and

$$
c=1.006 \nu[A] \nu\left[x^{(0)}\right]+1.004 n^{-1} \widetilde{\gamma} .
$$

Then

$$
\hat{r}=r+\delta r, \quad \nu[\delta r] \preccurlyeq \theta_{n} c .
$$

Proof. Let $r=\left(r_{1}, \cdots, r_{n}\right)^{t}$ and $s_{i}=\sum_{j=1}^{n} a_{i j} x_{j}^{(0)}$. Then we have

$$
\begin{aligned}
\tilde{r}_{i} & =f\left(\tilde{s}_{i}-b_{i}\right)=\tilde{s}_{i}-b_{i}+\left(\tilde{s}_{i}-b_{i}\right) \xi_{0} \\
& =\sum_{j=1}^{n} a_{i j} x_{j}^{(0)}\left(1+\xi_{j}\right)-b_{i}+\frac{\tilde{r}_{i}}{1+\xi_{0}} \xi_{0} \\
& =r_{i}+\delta r_{i} \quad(1 \leqq i \leqq n)
\end{aligned}
$$

where $i \xi_{0} \mid<\beta^{1-t}, \xi_{j}$ are defined in Lemma 2 and

$$
\delta r_{i}=\sum_{j=1}^{n} a_{i j} x_{j}^{(0)} \xi_{j}+\left(1+\xi_{0}\right)^{-1} \widetilde{r}_{i} \xi_{0} .
$$


Hence

$$
\begin{aligned}
\left|\delta r_{i}\right| & <1.006 \theta_{n} \sum_{j=1}^{n}\left|a_{i, j} x_{j}^{(0)}\right|+\left(1-\beta^{1-i}\right)^{-1} \widetilde{r}_{i} \beta^{1-\imath} \\
& <1.006 \theta_{n} \sum_{j=1}^{n}\left|a_{i j}\right| \cdot\left|x_{j}^{(0)}\right|+(1-0.0034)^{-1} \widetilde{r}_{i} n^{-1} \theta_{n} \\
& <c_{i} \theta_{n}
\end{aligned}
$$

where $c_{i}$ denotes the $i$-th component of the vector $c$ defined in (3.7). This implies $\nu[\delta r] \leqslant \theta_{n} c$ where $\delta r=\left(\delta r_{1}, \cdots, \delta r_{n}\right)^{\prime}$.

Q.E.D.

Lemma 4. Let $\varepsilon=\nu[L r]$ and

$$
d=\left(1.006\|\widetilde{r}\|_{\infty}+\|c\|_{\infty}\right) \nu[L](1, \cdots, 1)^{t} .
$$

Then

$$
\widetilde{\varepsilon}=\varepsilon+\delta \varepsilon, \quad \nu[\delta \varepsilon] \preccurlyeq \theta_{n} d .
$$

Proof. Let $\varepsilon_{i}$ and $d_{i}$ be the $i$-th components of the vectors $\varepsilon$ and $d$ respectively and set $L=\left(l_{i j}\right)$. Then we have from Lemma 2

$$
\begin{aligned}
\mathrm{fl}\left(\sum_{j=1}^{n} l_{i j} \widetilde{r}_{j}\right) & =\sum_{j=1}^{n} l_{i j} \widetilde{r}_{j}+\sum_{j=1}^{n} l_{i j} \widetilde{r}_{j} \xi_{j}\left(\left|\xi_{j}\right|<1.006 \theta_{n}\right) \\
& =\sum_{j=1}^{n} l_{i j}\left(r_{j}+\delta r_{j}\right)+\sum_{j=1}^{n} l_{i j} \widetilde{r}_{j} \xi_{j}
\end{aligned}
$$

so that

$$
\begin{aligned}
\left|\hat{\hat{s}}_{i}-\varepsilon_{i}\right| & \leqq|| \mathrm{fl}\left(\sum_{j=1}^{n} l_{i j} \widetilde{r}_{j}\right)|-| \sum_{i=1}^{n} l_{i, j} r_{j}|| \\
& \leqq\left|\sum_{j=1}^{n} l_{i j}\left(\delta r_{j}+\widetilde{r}_{j} \xi_{j}\right)\right| \\
& <\sum_{j=1}^{n}\left|l_{i j}\right|\left\{\theta_{n} c_{j}+\left|\widetilde{r}_{j}\right|\left(1.006 \theta_{n}\right)\right\} \leqq d_{i} \theta_{n} . \quad \text { Q.E.D. }
\end{aligned}
$$

Lemma 5. Let

$$
E=1.006 \nu[L] \nu[A]+1.004 n^{-1}\left(\begin{array}{lll}
\tilde{\kappa}_{11} & & \\
& \ddots & \\
& & \tilde{\kappa}_{n n}
\end{array}\right)
$$

Then ree have 


$$
\widetilde{K}=K+\delta K, \quad \nu[\delta K] \preccurlyeq \theta_{n} E .
$$

Proof. We denote the $(i, j)$ element of a matrix $M$ by $M_{i j}$, etc. Then we have

$$
\begin{aligned}
\left(I_{n}-\widetilde{L A}\right)_{i j} & =\delta_{i j}-\sum_{k=1}^{n} l_{i k} a_{k j}\left(1+\xi_{i k j}\right)\left(\left|\xi_{i k j}\right|<1.006 \theta_{n}\right) \\
& =\delta_{i j}-\sum_{j=1}^{n} l_{i k} a_{k j}-\sum_{k=1}^{n} l_{i k} a_{k j} \xi_{i k j} \\
& =\left(I_{n}-L A\right)_{i j}-\sum_{k=1}^{n} l_{i k} a_{k j} \xi_{i k j}
\end{aligned}
$$

where $\delta_{i j}$ denotes the Kronecker symbol. Therefore

$$
\begin{aligned}
\mathrm{fl}\left(I_{n}-\widetilde{L A}\right)_{i j} & =\left(I_{n}-\widetilde{L A}\right)_{i j}+\delta_{i j}\left(I_{n}-\widetilde{L A}\right)_{i i} \eta_{i} \quad\left(\left|\eta_{i}\right|<\beta^{1-t}\right) \\
& =\left(I_{n}-L A\right)_{i j}-\sum_{k=1}^{n} l_{i k} a_{k j} \xi_{i k j}+\delta_{i j}\left(I_{n}-\widetilde{L A}\right)_{i i} \eta_{i}
\end{aligned}
$$

so that we can write

$$
\widetilde{K}=K+\delta K
$$

where

$$
\begin{aligned}
\left|(\delta K)_{i j}\right| & \leqq \sum_{k=1}^{n}\left|l_{i k} a_{k j} \hat{\xi}_{i k j}\right|+\left|\hat{o}_{i j}\left(I_{n}-\widetilde{L A}\right)_{i i} \eta_{i}\right| \\
& <1.006 \theta_{n} \sum_{k=1}^{n}\left|l_{i k}\right|\left|a_{k j}\right|+\delta_{i j}\left(1-\beta^{1-t}\right)^{-1} \widetilde{K}_{i i} \beta^{1-t} \\
& <\theta_{n} E_{i j} .
\end{aligned}
$$

Lemma 6. Let $\kappa_{i}$ be defined as in the remark at the end of Section 2 and $e=\left(e_{1}, \cdots, e_{n}\right)^{t}$ where

$$
e_{i}=\sum_{j=1}^{n} E_{i j}+1.006 \sum_{j=1}^{n} \tilde{\kappa}_{i j} .
$$

Then we have

$$
\tilde{\kappa}=\kappa+\delta \kappa, \quad \nu[\delta \kappa] \preccurlyeq \theta_{n} e .
$$

Proof. We have

$$
\tilde{\kappa}_{i}=\mathrm{fl}\left(\tilde{\kappa}_{i 1}+\cdots+\tilde{\kappa}_{i n}\right)
$$




$$
\begin{aligned}
& =\sum_{j=1}^{n} \widetilde{\kappa}_{i j}+\sum_{j=1}^{n} \tilde{\kappa}_{i j} \xi_{j}\left(\left|\xi_{j}\right|<1.006 \theta_{n-1}\right) \\
& =\kappa_{i}+\delta \kappa_{i}
\end{aligned}
$$

where

$$
\delta \kappa_{i}=\sum_{j=1}^{n}(\delta K)_{i j}+\sum_{j=1}^{n} \tilde{\kappa}_{i j} \xi_{j}
$$

Hence

$$
\left|\delta \kappa_{i}\right| \leqq \sum_{j=1}^{n} E_{i j} \theta_{n}+1.006 \theta_{n-1} \sum_{j=1}^{n} \tilde{\kappa}_{i j}<\theta_{n} e_{i} \text { Q.E.D. }
$$

Lemma \%. Let.

$$
a=\frac{\|\varepsilon\|_{\infty}}{1-\|K\|_{\infty}}
$$

and

(3. 8) $f=1.004\left\{\frac{\|d\|_{\infty}+n^{-1}\|\tilde{\varepsilon}\|_{\infty}}{1-\|\tilde{\kappa}\|_{\infty}}+\frac{n^{-1}\|\varepsilon\|_{\infty}}{1-\|\kappa\|_{\infty}}+\frac{\|\varepsilon\|_{\infty}\|e\|_{\infty}}{\left(1-\|\tilde{\kappa}\|_{\infty}\right)\left(1-\|\kappa\|_{\infty}\right)}\right\}$.

Then we have

$$
\widetilde{a}=a+\delta a . \quad|\delta a|<f \theta_{n} .
$$

Proof. Let $\varepsilon=\left(\varepsilon_{1}, \cdots, \varepsilon_{n}\right)^{t}$

$$
\max _{i} \widetilde{\varepsilon}_{i}=\widetilde{\varepsilon}_{p} \text { and } \max _{i} \varepsilon_{i}=\varepsilon_{q} \text {. }
$$

Then

$$
\delta \varepsilon_{p} \geqq \delta \varepsilon_{p}+\left(\varepsilon_{p}-\varepsilon_{q}\right)=\widetilde{\varepsilon}_{p}-\varepsilon_{q} \geqq \delta \varepsilon_{q}
$$

so that

$$
\left|\widetilde{\varepsilon}_{p}-\varepsilon_{q}\right| \leqq \max \left(\left|\delta \varepsilon_{p}\right|,\left|\delta \varepsilon_{q}\right|\right) \leqq\|d\|_{\infty} \theta_{n} .
$$

Hence we can write $\widetilde{\varepsilon}_{p}=\varepsilon_{q}+\delta \varepsilon_{\infty}$ or

$$
\|\widetilde{\varepsilon}\|_{\infty}=\|\varepsilon\|_{\infty}+\delta \varepsilon_{\infty}
$$

where $\left|\delta \varepsilon_{\infty}\right| \leqq\|d\|_{\infty} \theta_{n}$. Similarly we have

$$
\|\tilde{\kappa}\|_{\infty}=\|\kappa\|_{\infty}+\delta \kappa_{\infty}
$$


where $\left|\delta \kappa_{\infty}\right| \leqq\|e\|_{\infty} \theta_{n}$. Therefore we have

$$
\begin{aligned}
\widetilde{a} & =\frac{\|\tilde{\varepsilon}\|_{\infty}}{\mathrm{fl}\left(1-\|\tilde{\kappa}\|_{\infty}\right)}\left(1+\xi_{1}\right) \quad\left(\left|\xi_{1}\right|<\beta^{1-t}\right) \\
& =\frac{\left(\|\varepsilon\|_{\infty}+\delta \varepsilon_{\infty}\right)\left(1+\xi_{1}\right)}{\left(1-\|\kappa\|_{\infty}-\delta \kappa_{\infty}\right)\left(1+\xi_{2}\right)} \quad\left(\left|\xi_{2}\right|<\beta^{1-t}\right)
\end{aligned}
$$

so that

$$
\widetilde{a}-a=\frac{\left(1-\|\kappa\|_{\infty}\right)\left(\delta \varepsilon_{\infty}+\|\tilde{\varepsilon}\|_{\infty} \xi_{1}\right)+\|\varepsilon\|_{\infty} \delta \kappa_{\infty}-\|\varepsilon\|_{\infty}\left(1-\|\tilde{\kappa}\|_{\infty}\right) \xi_{2}}{\left(1-\|\tilde{\kappa}\|_{\infty}\right)\left(1-\|\kappa\|_{\infty}\right)\left(1+\xi_{2}\right)} .
$$

It follows from this that

$$
|\widetilde{a}-a|<f \theta_{n}
$$

where $f$ is defined in (3.8).

Q.E.D.

We are now in a position to prove the following theorem.

Theorem 2. Let $\alpha$ be the vector defined in Theorem 1 with the maximum norm. Then, under the assumption of Theorem 1, we have

$$
\alpha \preccurlyeq \tilde{\alpha}+\delta \tilde{\alpha}
$$

where

$$
\delta \widetilde{\alpha}=\left\{1.004 n^{-1} \widetilde{\alpha}+d+a e+\left(f+\widetilde{a} n^{-1}\right) \widetilde{\kappa}\right\} \theta_{n}:
$$

Proof. We have

$$
\begin{aligned}
\widetilde{\alpha}_{i} & =\mathrm{fl}\left\{\widetilde{\varepsilon}_{i}+\mathrm{fl}\left(\widetilde{a} \tilde{\kappa}_{i}\right)\right\} \\
& =\left\{\tilde{\varepsilon}_{i}+\mathrm{fl}\left(\widetilde{a} \tilde{\kappa}_{i}\right)\right\}(1+\xi) \\
& =\varepsilon_{i}+\delta \varepsilon_{i}+(a+\delta a)\left(\kappa_{i}+\delta \kappa_{i}\right)(1+\eta)+\left\{\tilde{\varepsilon}_{i}+\mathrm{fl}\left(\widetilde{a} \tilde{\kappa}_{i}\right)\right\} \xi \\
& =\alpha \alpha_{i}+\delta \varepsilon_{i}+a \delta \kappa_{i}+\delta a \tilde{\kappa}_{i}+\widetilde{a} \tilde{\kappa}_{i} \eta+\left\{\tilde{\varepsilon}_{i}+\mathrm{fl}\left(\widetilde{a} \tilde{\kappa}_{i}\right)\right\} \xi
\end{aligned}
$$

where $|\xi|,|\eta|<\beta^{1-t}$. Hence

$$
\begin{aligned}
\left|\tilde{\alpha}_{i}-\alpha_{i}\right| & <d_{i} \theta_{n}+a e_{i} \theta_{n}+\left(f \theta_{n}\right) \tilde{\kappa}_{i}+\widetilde{a} \tilde{\kappa}_{i} \beta^{1-t}+\left(1-\beta^{1-t}\right)^{-1} \tilde{\alpha}_{i} \beta^{1-t} \\
& <\left\{d_{i}+a e_{i}+f \tilde{\kappa}_{i}+\widetilde{a} \tilde{\kappa}_{i} n^{-1}+1.004 \tilde{\alpha}_{i} n^{-1}\right\} \theta_{n}
\end{aligned}
$$

which means 


$$
\alpha \preccurlyeq \tilde{\alpha}+\delta \alpha
$$

where $\delta \alpha$ is defined by (3.9).

Q.E.D.

We have from Lemmas 3-7 that

$$
\begin{aligned}
d_{i} \leqq & \left(1.006\|\tilde{r}\|_{\infty}+\|c\|_{\infty}\right)\|L\|_{\infty} \\
& <\left[\left(1.006+1.004 n^{-1}\right)\|\tilde{r}\|_{\infty}+1.006\|A\|_{\infty}\left\|x^{(0)}\right\|_{\infty}\right]\|L\|_{\infty}
\end{aligned}
$$

and

$$
\begin{aligned}
e_{i} & <\|E\|_{\infty}+1.006\left(1-1.006 \theta_{n-1}\right)^{-1} \mathrm{fl}\left(\tilde{\kappa}_{i 1}+\cdots+\tilde{\kappa}_{i n}\right) \\
& <1.006\|L\|_{\infty}\|A\|_{\infty}+1.004 n^{-1} \max _{i} \tilde{\kappa}_{i i}+1.02\|\tilde{\kappa}\|_{\infty} .
\end{aligned}
$$

Therefore, we can say that, if $\|\tilde{\kappa}\|_{\infty} \ll 1$ and

$$
\|L\|_{\infty}\left(\|A\|_{\infty}+\|A\|_{\infty}\left\|x^{(0)}\right\|_{\infty}+\|\widetilde{r}\|_{\infty}\right) \theta_{n}
$$

is small enough, then each component of the vector $\delta \alpha$ is small as compared with that of $\alpha$ and our method works well. Observe also that, for our purpose, we need not know the exact $\delta \alpha$. It suffices to know the order of each component. Hence, in practice, the following result may be useful:

Theorem 3. Let

$$
\begin{aligned}
& \tilde{A}_{\infty}=\max _{i} \mathrm{fl}\left(\left|a_{i 1}\right|+\cdots+\left|a_{i n}\right|\right), \\
& \widetilde{L}_{\infty}=\max _{i} \mathrm{fl}\left(\left|l_{i 1}\right|+\cdots+\left|l_{i n}\right|\right), \\
& \tilde{\kappa}_{\infty}=\max _{i} \mathrm{fl}\left(\tilde{\kappa}_{i 1}+\cdots+\tilde{\kappa}_{i n}\right) \quad\left(=\|\tilde{\kappa}\|_{\infty}\right), \\
& \tilde{c}_{\infty}=1.02 \tilde{A}_{\infty}\left\|x^{(0)}\right\|_{\infty}+0.502\|\tilde{r}\|_{\infty}, \\
& \tilde{d}_{\infty}=1.02 \widetilde{L}_{\infty}\left(\|\tilde{r}\|_{\infty}+\tilde{c}_{\infty}\right),
\end{aligned}
$$

and

$$
\tilde{e}_{\infty}=1.03\left(\widetilde{L}_{m} \tilde{A}_{\infty}+\widetilde{\kappa}_{\infty}\right)+0.502 \max \tilde{\kappa}_{i i}
$$

Further, assume that $\tilde{e}_{\infty} \theta_{n}<1$ and there exists a positive number $m$ such that $\tilde{\kappa}_{\infty}<1-m^{-1}-\tilde{e}_{\infty} \theta_{n}$. Set

$$
\tilde{f}_{\infty}=1.004(m-1)\left\{\left(1+n^{-1}+m \tilde{e}_{\infty} \theta_{n}\right) \tilde{d}_{\infty}+\left(2 n^{-1}+m \tilde{e}_{\infty}\right)\|\widetilde{\varepsilon}\|_{\infty}\right\}
$$


and

$$
\Delta \tilde{\alpha}_{i}=1.004 \beta^{1-t} \tilde{\alpha}_{i}+\left\{\tilde{d}_{\infty}\left(1+m \tilde{e}_{\infty} \theta_{n}\right)+m\|\widetilde{\varepsilon}\|_{\infty} \tilde{e}_{\infty}+\tilde{f}_{\infty}+n^{-1} \widetilde{a} \tilde{\kappa}_{\infty}\right\} \theta_{n} .
$$

Then

$$
\left|x_{i}^{*}-x_{i}^{(0)}\right| \leqq \tilde{\alpha}_{i}+\Delta \tilde{\alpha}_{i}, \quad i=1,2, \cdots, n \text {. }
$$

Proof. We obtain from Theorem 2,

$$
\begin{aligned}
\|c\|_{\infty} & \leqq 1.006\|A\|_{\infty}\left\|x^{(0)}\right\|_{\infty}+1.004 n^{-1}\|\widetilde{r}\|_{\infty} \\
& <1.006\left(1-1.006 \theta_{n-1}\right)^{-1} \tilde{A}_{\infty}\left\|x^{(0)}\right\|_{\infty}<\tilde{c}_{\infty}, \\
\|d\|_{\infty} & \leqq\|L\|_{\infty}\left(1.006\|r\|_{\infty}+\|c\|_{\infty}\right)<1.02 \widetilde{L}_{\infty}\|\widetilde{r}\|_{\infty}+1.011 \tilde{c}_{\infty} \\
& <\tilde{d}_{\infty}
\end{aligned}
$$

and

$$
\begin{aligned}
\|e\|_{\infty} & <\|E\|_{\infty}+1.006\left(1-1.006 \theta_{n-1}\right)^{-1} \tilde{\kappa}_{i} \\
& <1.006\|L\|_{\infty}\|A\|_{\infty}+1.004 n^{-1} \max _{i} \widetilde{\kappa}_{i i}+1.02 \widetilde{\kappa}_{i} \\
& <1.006(1-0.01)^{-1} \widetilde{L}_{\infty}(1-0.01)^{-1} \tilde{A}_{\infty}+0.502 \max _{i} \tilde{\kappa}_{i i}+1.02 \widetilde{\kappa}_{i} \\
& <\widetilde{e}_{\infty} .
\end{aligned}
$$

Moreover, if $\widetilde{\kappa}_{\infty}<1-m^{-1}-\tilde{e}_{\infty} \theta_{n}$ for some $m>0$, then we have

$$
\left(1-\|\tilde{\kappa}\|_{\infty}\right)^{-1}\|\tilde{\kappa}\|_{\infty}<m-1,\left(1-\|\kappa\|_{\infty}\right)^{-1}<\left(1-\|\tilde{\kappa}\|_{\infty}-\tilde{e}_{\infty} \theta_{n}\right)^{-1}<m
$$

and

$$
\left(1-\|\kappa\|_{\infty}\right)^{-1} \tilde{\kappa}_{\infty}<m-1
$$

Hence

$$
a e_{i} \leqq m\|\varepsilon\|_{\infty} \widetilde{e}_{\infty} \leqq m\left(\|\widetilde{\varepsilon}\|_{\infty}+\tilde{d}_{\infty} \theta_{n}\right) \tilde{e}_{\infty}
$$

and

$$
\begin{aligned}
& f \tilde{\kappa}_{i} \leqq f\|\tilde{\kappa}\|_{\infty}<1.004(m-1)\left\{\tilde{d}_{\infty}+n^{-1}\|\tilde{\varepsilon}\|_{\infty}+n^{-1}\|\varepsilon\|_{\infty}+m\|\varepsilon\|_{\infty} \tilde{e}_{\infty}\right\} \\
& \quad<1.004(m-1)\left\{\tilde{d}_{\infty}+n^{-1}\|\tilde{\varepsilon}\|_{\infty}+\left(n^{-1}+m \tilde{e}_{\infty}\right)\left(\|\tilde{\varepsilon}\|_{\infty}+\tilde{d}_{\infty} \theta_{n}\right)\right\}=\tilde{f}_{\infty} .
\end{aligned}
$$

The result now follows from Theorem 2 .

Q.E.D. 


\section{§4. Namerical Examples}

We shall illustrate our results by simple examples.

Example 1. Consider the linear system $A x=b$ given by

$$
\begin{aligned}
& 0.51273 x_{1}+0.62137 x_{2}=0.14012 \\
& 0.41835 x_{1}+0.50701 x_{2}=0.34827
\end{aligned}
$$

which is due to Peters and Wilkinson [4]. As is remarked there, this is extremely ill-conditioned and has the exact solution vector $x^{*}=(-15977$. $7406 \cdots, 13184.4264 \cdots)^{t}$. We solve this system by Gaussian ellimination. A single precision computation (chopping the results to 6 hexadecimal digits in the mantissa) on FACOM 230-28 computer of Ehime University yields

$$
x^{(0)}=(-15594.90,12868.53)^{t} .
$$

The matrix $L$, a numerical result for $A^{-1}$, is also given by

$$
L=\left(\begin{array}{rr}
0.5439359 E+5 & -0.6666244 E+5 \\
-0.4488187 E+5 & 0.5500726 E+5
\end{array}\right) \text {. }
$$

We then compute $K=\nu\left[I_{2}-L A\right], \varepsilon=\nu\left[L\left(A x^{(0)}-b\right)\right]$ and $\alpha$, etc., with double precision arithmetic (chopping the results to 14 hexadecimal digits in the mantissa). Then $\|\tilde{\kappa}\|_{\infty}\left(=\widetilde{\kappa}_{\infty}\right)=0.028 \cdots<1$ and Theorem 1 is applicable. The results are shown in Table 1.

Table 1. Error bounds for $x^{(0)}$ given by (4.1).

\begin{tabular}{cccrr}
\hline$i$ & $\tilde{\alpha}$ & $\tilde{\alpha}^{(1)}$ & \multicolumn{1}{c}{$\tilde{\varepsilon}$} & \multicolumn{1}{c}{$\tilde{\gamma}$} \\
\hline 1 & $384.5585 \ldots$ & $382.8805 \ldots$ & $373.669 \ldots$ & $0.686 \ldots \mathrm{E}-2$ \\
2 & $317.2004 \ldots$ & $315.9270 \ldots$ & $308.326 \ldots$ & $-0.226 \ldots \mathrm{E}-5$ \\
\hline
\end{tabular}

Next, we apply Theorem 3 to estimate the effect of the errors made in the computation. Observe that, in our computer, $\beta=16$ and $t=14$. Then we have

$$
\tilde{d}_{\infty}=0.558 \cdots E+5, \tilde{e}_{\infty}=0.141 \cdots E+6, \tilde{\kappa}_{\circ 2}<0.5-\tilde{e}_{\infty} \theta_{n}, \text { etc., }
$$

so that we take $m=2$ for simplicity to compute $f_{\infty}$ and obtain

$$
\tilde{f}_{\infty}=0.74 \cdots E+5 \text { and }\|\Delta \tilde{\alpha}\|_{\infty}=0.252 \cdots E-5<0.253 E-5 \text {. }
$$


This implies that

$$
\nu\left[x^{*}-x^{(0)}\right] \preccurlyeq \tilde{\alpha}+0.253 E-5\left(\begin{array}{l}
1 \\
1
\end{array}\right) \preccurlyeq\left(\begin{array}{l}
384.5586 \\
317.2005
\end{array}\right),
$$

or

$$
\left(\begin{array}{r}
-15979.46 \\
12551.32
\end{array}\right) \preccurlyeq x^{*} \preccurlyeq\left(\begin{array}{r}
-15210.35 \\
13185.74
\end{array}\right)
$$

On the other hand, if we use the double precision arithmetic to compute $x^{(0)}$ and $L$, then we have

$$
\begin{aligned}
& x^{(0)}=(-15977.74063 \cdots, 13184.42647 \cdots)^{t}, \\
& L=\left(\begin{array}{rr}
0.557288 \cdots E+5 & -0.682989 \cdots E+5 \\
-0.459836 \cdots E+5 & 0.563575 \cdots E+5
\end{array}\right),
\end{aligned}
$$

and

$$
\tilde{\kappa}_{\infty}=0.109 \cdots E-10 .
$$

The large change of $x^{(0)}$ from (4.1) to (4.2) (as well as $L$ ) reflects the ill-conditionality of the system. The results of Theorem 1 applied to $x^{(0)}$ given by $(4.2)$ and the above $L$ are shown in Table 2.

Table 2. Error bounds for $x^{(0)}$ given by (4.2).

\begin{tabular}{ccccc}
\hline$i$ & $\tilde{\alpha}$ & $\tilde{\alpha}^{(1)}$ & $\tilde{\varepsilon}$ & $\tilde{r}$ \\
\hline 1 & $0.392526 \ldots \mathrm{E}-7$ & $0.392526 \ldots \mathrm{E}-7$ & $0.392526 \ldots \mathrm{E}-7$ & $0.181 \ldots \mathrm{E}-11$ \\
2 & $0.323868 \ldots \mathrm{E}-7$ & $0.323868 \ldots \mathrm{E}-7$ & $0.323868 \ldots \mathrm{E}-7$ & $0.909 \ldots \mathrm{E}-12$ \\
\hline
\end{tabular}

In this case, we have $\tilde{e}_{\infty}=0.144 \cdots E+6$ and again take $m=2$ to compute $f_{\infty}$. Then we obtain $\|\Delta \widetilde{\alpha}\|_{\infty}=0.260 \cdots E-5$ (which is larger than that of the single precision arithmetic). Thus we can assert that

$$
\left(\begin{array}{r}
-15977.74064 \\
13184.42646
\end{array}\right) \preccurlyeq x^{*} \preccurlyeq\left(\begin{array}{r}
-15977.74062 \\
13184.42648
\end{array}\right) .
$$

Example 2. Consider the linear system given by

$$
\begin{aligned}
& 0.876543 x_{1}+0.617341 x_{2}+0.589973 x_{3}=0.863257 \\
& 0.612314 x_{1}+0.784461 x_{2}+0.827742 x_{3}=0.820647 \\
& 0.317321 x_{1}+0.446779 x_{2}+0.476349 x_{3}=0.450098
\end{aligned}
$$


which is found in Wilkinson [7] and is discussed also in Yamamoto [9]. This system is ill-conditioned, too. We again solve this by Gaussian ellimination with single precision arithmetic. Then we obtain a numerical solution

$$
x^{(0)}=(0.6363233,-0.2946413 E-1,0.5486381)^{t} .
$$

At the same time, we have a matrix $L$, approximation for $A^{-1}$, such that $\widetilde{L}_{\infty}=0.657 \cdots E+5$ (see Yamamoto [9]). In this case, by the double precision computation, we have

$$
\tilde{\kappa}_{\infty}=0.967 \cdots E-2 \text { and } \tilde{e}_{\infty}=0.150 \cdots E+6 .
$$

The vectors $\tilde{\alpha}, \widetilde{\alpha}^{(1)}, \widetilde{\varepsilon}$ and $\widetilde{r}$ are shown in Table 3 .

Table 3. Error bounds for $x^{(0)}$ given by (4.3).

\begin{tabular}{ccccc}
\hline$i$ & $\tilde{\alpha}$ & $\tilde{\alpha}^{(1)}$ & $\tilde{\varepsilon}$ & $\tilde{r}$ \\
\hline 1 & $0.573591 \ldots \mathrm{E}-5$ & $0.570495 \ldots \mathrm{E}-5$ & $0.560957 \ldots \mathrm{E}-5$ & $0.192 \ldots \mathrm{E}-7$ \\
2 & $0.427810 \ldots \mathrm{E}-4$ & $0.426081 \ldots \mathrm{E}-4$ & $0.423670 \ldots \mathrm{E}-4$ & $0.303 \ldots \mathrm{E}-7$ \\
3 & $0.362315 \ldots \mathrm{E}-4$ & $0.361321 \ldots \mathrm{E}-4$ & $0.359655 \ldots \mathrm{E}-4$ & $0.165 \ldots \mathrm{E}-7$ \\
\hline
\end{tabular}

Further, if we apply Theorem 3 by taking $m=2$, then we have

$$
\Delta \widetilde{\alpha}_{i}=0.150 \cdots E-1<0.151 E-9
$$

which implies that

$$
\nu\left[x^{*}-x^{(0)}\right] \preccurlyeq \tilde{\alpha}^{(0)}+0.151 E-9\left(\begin{array}{l}
1 \\
1 \\
1
\end{array}\right) \preccurlyeq\left(\begin{array}{l}
0.573607 E-5 \\
0.427813 E-4 \\
0.362317 E-4
\end{array}\right),
$$

or

$$
\left(\begin{array}{c}
0.6363177 \\
-0.295069 E-1 \\
0.5486019
\end{array}\right) \preccurlyeq x^{*} \preccurlyeq\left(\begin{array}{c}
0.6363291 \\
-0.2942135 E-1 \\
0.5486743
\end{array}\right)
$$

If we compute $x^{(0)}$ and $L$ by the double precision arithmetic, then we have

(4. 4) $\quad x^{(0)}=(0.63632896 \cdots,-0.29506656 \cdots E-1,0.54867420 \cdots)^{t}$, and

$$
\widetilde{L}_{\infty}=0.66 \cdots E+5
$$


The double precision computation yields $\tilde{\kappa}_{\infty}=0.272 \cdots E-11$ and $\tilde{e}_{\infty}=0.151$ $\cdots E+6$. The vectors $\widetilde{\alpha}$ and $\widetilde{\alpha}^{(1)}$, etc., are shown in Table 4.

Table 4. Error bounds for $x^{(0)}$ given by (4.4).

\begin{tabular}{ccccr}
\hline$i$ & $\tilde{\alpha}$ & $\tilde{\alpha}^{(1)}$ & $\tilde{\varepsilon}$ & \multicolumn{1}{r}{} \\
\hline 1 & $0.157211 \ldots \mathrm{E}-14$ & $0.157211 \ldots \mathrm{E}-14$ & $0.157 \ldots \mathrm{E}-14$ & $-0.138 \ldots \mathrm{E}-16$ \\
2 & $0.126332 \ldots \mathrm{E}-13$ & $0.126332 \ldots \mathrm{E}-13$ & $0.126 \ldots \mathrm{E}-13$ & $0.416 \ldots \mathrm{E}-16$ \\
3 & $0.108600 \ldots \mathrm{E}-13$ & $0.108600 \ldots \mathrm{E}-13$ & $0.108 \ldots \mathrm{E}-13$ & $0.277 \ldots \mathrm{E}-16$ \\
\hline
\end{tabular}

Further we have

$$
\Delta \widetilde{\alpha}_{i}=0.151 \cdots E-9
$$

where we have taken $m=2$ to compute $f_{\infty}$. Thus we obtain

$$
\nu\left[x^{*}-x^{(0)}\right] \preccurlyeq \widetilde{\alpha}+0.152 E-9\left(\begin{array}{l}
1 \\
1
\end{array}\right) \preccurlyeq 0.153 E-9\left(\begin{array}{l}
1 \\
1
\end{array}\right) .
$$

Example 3. Consider the linear system

$$
\begin{aligned}
& 33 x_{1}+16 x_{2}+72 x_{3}=152.833 \\
& -24 x_{1}-10 x_{2}-57 x_{3}=-94.324 \\
& -8 x_{1}-4 x_{2}-17 x_{3}=-38.308
\end{aligned}
$$

which has the exact solution $x^{*}=(-0.001,10,-0.1)^{t}$. Then, by the single precision computation, we obtain

$$
x^{(0)}=(0.1018889 E-2,0.9999983 E+1,-0.1000051)^{t}
$$

and

$$
L=\left(\begin{array}{rrr}
-9.667 \cdots & -2.666 \cdots & -32.001 \cdots \\
8.003 \cdots & 2.500 \cdots & 25.501 \cdots \\
2.666 \cdots & 0.666 \cdots & 9.000 \cdots
\end{array}\right) \text {, }
$$

so that the system is well-conditioned. The double precision computation yields

$$
\tilde{\kappa}_{\infty}=0.213 \cdots E-3
$$

and the vector $\widetilde{\alpha}, \widetilde{\alpha}^{(1)}$ and $\widetilde{\varepsilon}$, etc., are shown in Table 5. Further we have from Theorem 3

$$
\|\Delta \widetilde{\alpha}\|_{\infty}=0.869 \cdots E-10<0.87 E-10 .
$$


Table 5. Error bounds for $x^{(0)}$ given by (4.5).

\begin{tabular}{|c|c|c|c|c|}
\hline$i$ & $\tilde{\alpha}$ & $\tilde{\alpha}^{(1)}$ & $\tilde{\varepsilon}$ & $\tilde{r}$ \\
\hline 1 & $0.188865 \ldots \mathrm{E}-4$ & $0.188848 \ldots \mathrm{E}-4$ & $0.188825 \ldots \mathrm{E}-4$ & $-0.223 \ldots \mathrm{E}-4$ \\
\hline 2 & $0.171678 \cdots \mathrm{E}-4$ & $0.171674 \ldots \mathrm{E}-4$ & $0.171667 \ldots \mathrm{E}-4$ & $0.120 \ldots \mathrm{E}-4$ \\
\hline 3 & $0.515085 \ldots \mathrm{E}-5$ & $0.515052 \ldots \mathrm{E}-5$ & $0.515004 \ldots \mathrm{E}-5$ & $0.515 \ldots \mathrm{E}-5$ \\
\hline
\end{tabular}

Therefore, our method works well in this case, too.

\section{References}

[1] Collatz, L., Functional analysis and numerical mathematics, Academic Press, New York, 1966.

[2] Forsythe, G. and Moler, C., Computer solution of linear algebraic systems, PrenticeHall, Englewood Cliffs, New Jersey, 1967.

[3] Paige, C. C., Error analysis of the symmetric Lanczos process for the eigenproblem, London Univ. Inst. of Computer Science, Tech. Note ICSI, 209, 1969.

[4] Peters, G. and Wilkinson, J. H., Inverse iteration, ill-conditioned equations and Newton method, SIAM Rev., 21 (1979), 339-360.

[5] Urabe, M., A posteriori component-wise error estimation of approximate solutions to nonlinear equations, Lecture Notes in Computer Sci., 29, Springer, New York, 1975.

[6] Wilkinson, J. H., Rounding errors in algebraic processes, Prentice-Hall, Englewood Cliffs, New Jersey, 1963.

[7] - The algebraic eigenvalue problem, Oxford Univ. Press, London, 1965.

[8] Yamamoto, T., Error bounds for computed eigenvalues and eigenvectors, Numer. Math., 34 (1980), 189-199.

[9] — Error bounds for approximate solutions of systems of equations, to appear.

[10] Componentwise error estimates for approximate solutions of systems of equations, Lecture Notes in Num. Appl. Anal., 3 (1981), 1-22. 
